\title{
ESTIMATION OF SALIVARY MATRIX METALLOPROTEINASE-9 IN ORAL LEUKOPLAKIA, ORAL SUBMUCOUS FIBROSIS, AND HEALTHY INDIVIDUALS: A COMPARATIVE OBSERVATIONAL STUDY
}

\author{
Akshaya Thiruvalluvan', Jagat R.C. Reddy', Vandana Sekizhar', Veni Subramanyam², Sivasankari T. ${ }^{1}$, Varsha V. ${ }^{2}$ \\ 'Department of Oral Medicine and Radiology, Indira Gandhi Institute of Dental Sciences, Sri Balaji Vidyapeeth (Deemed to be university), Puducherry, India \\ ${ }^{2}$ Central Inter-Disciplinary Research Facility (CIDRF), Sri Balaji Vidyapeeth, Pillaiyarkuppam, Puducherry, India
}

\begin{abstract}
INTRODUCTION: Salivary matrix metalloproteinase-9 (MMP-9) is an established diagnostic biomarker for detection and prognosis of oral cancer.

ОвJECTIVES: The current study aimed to estimate salivary MMP-9 in oral leukoplakia and oral submucous fibrosis (OSMF), which are considered as oral potentially malignant disorders (OPMDs).

MATERIAL AND METHODS: A comparative observational study of 60 subjects was conducted, including 20 patients diagnosed with OSMF (group A, $n=20$ ), 20 diagnosed with oral leukoplakia (group B, $n=20$ ), and 20 as healthy controls (group C, $n=20$ ) according to standard criteria. Unstimulated salivary samples were collected for evaluation of MMP-9 by enzyme-linked immunosorbent assay (ELISA). One-way analysis of variance (ANOVA) test was applied for comparison of groups. Bonferroni-adjusted one-way ANOVA test was performed for intra-group comparison.

RESULTS: Descriptive statistics of the data revealed that mean age of study participants in group A, group B, and group C were $50.30 \pm 9.96$ years, $46.70 \pm 12.59$ years, and $33.30 \pm 4.70$ years, respectively. Mean salivary MMP-9 level in OSMF, oral leukoplakia, and control group were $9.42 \mathrm{ng} / \mathrm{ml}, 10.59 \mathrm{ng} / \mathrm{ml}$, and $2.96 \mathrm{ng} / \mathrm{ml}$, respectively, with a $p$-value $<0.001$ between OSMF and oral leukoplakia when compared with controls.

ConcLusıons: A significant difference was noted in salivary MMP-9 levels among patients with OPMD's compared to controls. A slightly higher value of MMP-9 was observed in oral leukoplakia. Estimation of salivary MMP-9 level is a non-invasive and a reliable biomarker in diagnosis of OPMD's and in accessing their malignant transformation.
\end{abstract}

KEY WORDS: matrix metalloproteinase-9, oral potentially malignant disorders, oral leukoplakia, oral submucous fibrosis, oral squamous cell carcinoma.

J Stoma 2021; 74, 4: 221-226

DOI: https://doi.org/10.5114/jos.2021.111644

\section{INTRODUCTION}

Oral potentially malignant disorders (OPMD's) are the most common potential malignant disorders of the oral mucosa, which pose an increased risk of malignant transformation when compared to normal mucosa [1]. The World Health Organization (WHO) defined OPMD as clinical presentations that carry a risk of cancer development in the oral cavity, whether in a clinically definable precursor lesion or in clinically normal oral mucosa [2].

\section{JOURNAL OF STOMATOLOGY CZASOPISMO STOMATOLOGICZNE

ADDRESS FOR CORRESPONDENCE: Akshaya Thiruvalluvan, Department of Oral Medicine and Radiology, Indira Gandhi Institute of Dental Sciences, Sri Balaji Vidyapeeth (Deemed to be university), Pillaiyarkuppam, Pondicherry 607402, India, e-mail: akshaya.thiruvalluvan@gmail.com ReCeIVED: 21.04.2021 • ACCEPTED: 12.06.2021 • Published: 30.11.2021 
OPMD's are of concern in this study, being oral leukoplakia and oral submucous fibrosis (OSMF). However, other OPMD's include erythroplakia, proliferative verrucous leukoplakia (PVL), reverse smokers palate, dyskeratosis congenita, lichen planus, cheilitis glandularis, and xeroderma pigmentosum.

Leukoplakia and OSMF are the most common OPMDs that are linked to oral cancer [3]. The incidence of oral leukoplakia transforming to malignancy was estimated as $2.6 \%$ globally, ranging between $0.2-2.3 \%$ in Indian males and $1.2-4.6 \%$ in Indian females. Likewise, the global incidence for malignant transformation of OSMF ranges between $1.5-15 \%$, with $0.2-2.3 \%$ in Indian males and $1.2-4.6 \%$ in Indian females; in oral leukoplakia, it was estimated to be $0.1-17.5 \%$ [4-7]. It is an established fact that $50 \%$ of oral squamous cell carcinomas arises from OPMD's, and detection of oral squamous cell carcinoma (OSCC) in advanced stages results in a low survival rates.

Early diagnosis would lead to improved outcomes, and is predicted to increase five-year survival rate to around $80 \%$ [8]. OSCC, if detected in early (T1 stage) or in its' pre-cancerous stage (PMD), the 5-year survival is as high as $80 \%$, whereas if it is detected in late stages (T3 and T4 stages), survival rates are as low as $20-40 \%$ [9]. Moreover, a delay in the diagnosis of OPMD is the commonest cause for malignant transformation.

Early diagnosis of OPMDs is achieved by testing for variety of diagnostic markers, of which non-invasive salivary biomarkers offer promising results $[7,8]$. Tumor biomarkers can be used for an assessment of cancer patients or as prognostic indicators of neoplastic process. Matrix metalloproteinase-9 (MMP-9) is one tumor biomarker that belongs to extracellular zinc-dependent endopeptidases family and has the capability of degrading different components of extra cellular matrix (ECM) and non-matrix proteins [10]. MMPs have a role to control initial stages of carcinogenesis and their invasion, and serve as mediators for alterations in carcinogenesis [10]. Salivary biomarkers, including MMP-9, have been known for their role in proteolytic disintegration of basement membrane by specifically targeting collagens V, VII, and $\mathrm{X}$, fibronectin, elastin and type IV collagen meshwork. This is regarded to be a crucial step that encourages the invasion of tumors and metastasis [10].

\section{OBJECTIVES}

On reviewing literature, there are many studies related to MMP-9 and OSCC. However, studies between MMP-9 as a salivary biomarker and OPMD's (leukoplakia and OSMF) are a mear meagre, and paved a way for the current study. It was designed to establish a possible influence of salivary MMP-9 in the malignant transformation of OSMF and oral leukoplakia, both of which are a lead cause of OSCCs in Indian population.

\section{MATERIAL AND METHODS}

The study was designed to be a comparative observational study conducted in the Department of Oral Medicine and Radiology, Indira Gandhi Institute of Dental Sciences, Sri Balaji Vidyapeeth (Deemed to be university), Puducherry, in collaboration with the Central Interdisciplinary Research Facility (CIDRF), Sri Balaji Vidyapeeth, Pillaiyarkuppam, Puducherry, from May, 3, 2019 to February, 25, 2020.

The study was approved by institutional ethical clearance (IEC) prior to its' start (reference number: IGIDSIEC2018NRP30PGAKOMR), and reviewed by institutional review board (IRB). The study was registered with Clinical Trials Registry of India (reference number: CTRI/2020/06/026102) for recruitment of participants. A written informed consent was obtained from all participants upon fulfilling inclusion criteria.

The sample size was determined with significance level $\alpha=0.05$ and power $=90 \%$ to be 60 patients $(20$ in each of the three investigated groups), using the following formula: $n \geq\left[Z_{1-\alpha / 2}^{2} \times p(1-p)\right] / d^{2}$. The sampling method employed was systematic sampling, i.e., every case reporting to dental outpatient department (OPD) was recruited if found suitable as per the criteria.

Subjects with clinically and histopathologically diagnosed OSMF and oral leukoplakia, between age group of 19-75 years were included in group A and group B, respectively. The participants who were previously treated for OPMD's, OSCC, patients with psychological disorders and other systemic diseases, pregnant or lactating mothers, and patients under steroid therapy, were all excluded from the study.

Oral leukoplakia is a white patch, diagnosed by exclusion of any other similar lesion on clinically/histological grounds, but for its' association with use of tobacco products [4]. The WHO stated it to be 'a white patch or plaque that cannot be characterized clinically or histologically as any other disease', and clinically, it cannot be removed by simple scraping, thus differentiating form other common disorders or candida infection. The landmark histological features of oral dysplasia are concurrent with clinical leukoplakia. These include increased cell and nuclear volume, pleomorphism hyperplasia of basal cells, prominent enlarged nucleoli with hyperchromatic nuclei, increased nuclear to cytoplasmic ratio, keratinization of independent cell, etc. Clino-histological correlation, along with exclusion of possible differential diagnoses, aids in identification of oral leukoplakia as described previously [4].

The nature of the present study was explained to the participants, and they were reassured regarding maintenance of confidentiality. All the participants were assessed by one investigator, who was a qualified oral medicine specialist with a experience of 11 years. Demographic data and detailed case history were recorded. The study participants were divided into three groups, including group A: OSMF, group B: oral leukoplakia, and group C: controls. 
Saliva samples were collected in the morning between 8:00-10:00 am, and the subjects were instructed to refrain from eating, drinking, or oral hygiene procedures for at least an hour prior sample collection. Then they were given distilled drinking water and asked to rinse their mouth for 1 minute. Five minutes after oral rinse, they were seated quietly with the head bent down and the mouth open. This allowed the saliva to drip passively from the lower lip into the graduated sterile centrifuge tube, and about $5 \mu \mathrm{l}$ of saliva was collected and stored in ice box for transportation. Samples were then stored in sterile Eppendorf tubes (AG-22331; Hamburg, Germany). About $5 \mu \mathrm{l}$ of obtained un-stimulated saliva was collected and centrifuged at $10,000 \mathrm{~g}$ at $4^{\circ} \mathrm{C}$ for 2 minutes, and stored at $-80^{\circ} \mathrm{C}$. MMP-9 levels were measured in the samples using human MMP-9 ELISA kit (human matrix metalloproteinase 9 ELISA kit, Elabscience Immunodiagnostic Technologies, Texas, USA). Human MMP-9 ELISA kit applies to in vitro quantitative determination of human MMP-9 concentration in serum and plasma at a sensitivity of $0.1 \mathrm{ng} / \mathrm{ml}$, and a detection range of $0.16-10 \mathrm{ng} / \mathrm{ml}$, with repeatability of coefficient of variation as $<10 \%$.

\section{RESULTS}

Descriptive statistics revealed the mean age of the study participants in group $\mathrm{A}$, group $\mathrm{B}$, and group $\mathrm{C}$ were $50.30 \pm 9.96$ years, $46.70 \pm 12.59$ years, and $33.30 \pm 4.70$ years, respectively (Figure 1). The majority of the patients in group A were aged between 40 and 49 years (35.0\%). The subjects in group B were also aged between 40 and 49 years (35.0\%). Group C consisted of patients between 30 and 39 years of age (50.0\%). In group A, $60.0 \%$ were males and $40.0 \%$ were females. Among group B, $95.0 \%$ were males and $5.0 \%$ were females. In group C, $75.0 \%$ were males and $25.0 \%$ were females (Table 1 ).

The mean value of MMP-9 level in group A, group B, and group $\mathrm{C}$ was $9.42 \pm 2.882 \mathrm{ng} / \mathrm{ml}, 10.59 \pm 2.862 \mathrm{ng} / \mathrm{ml}$,

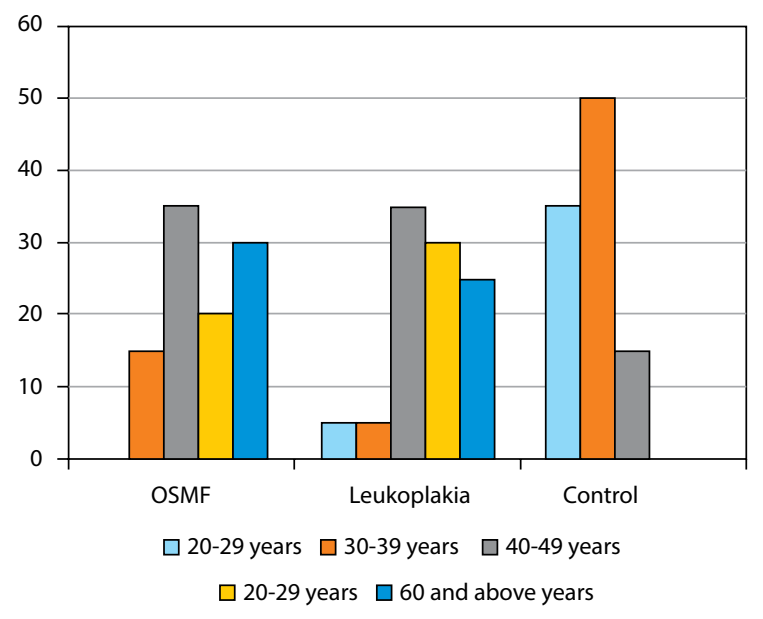

FIGURE 1. Age distribution of the study participants
TABLE 1. Demographics and habit-related data amongst study groups

\begin{tabular}{|c|c|c|}
\hline Demographics and habit data & $\begin{array}{l}\text { Group A } \\
\text { (osMF, } \\
n=20)\end{array}$ & $\begin{array}{c}\text { Group B } \\
\text { (oral leukoplaki } \\
n=20 \text { ) }\end{array}$ \\
\hline Mean age (years) & $50.30 \pm 9.96$ & $46.70 \pm 12.59$ \\
\hline Sex ratio (male : female) & $3: 2$ & $19: 1$ \\
\hline \multicolumn{3}{|l|}{ Type of habit, percentage (frequency) } \\
\hline Smoking tobacco & $0 \%$ & $n=10$ \\
\hline Smokeless tobacco (betel quid) & $80 \%(n=16)$ & $35 \%(n=7)$ \\
\hline Combined & $20 \%(n=4)$ & $15 \%(n=3)$ \\
\hline \multicolumn{3}{|c|}{ Age of onset of habit, percentage (frequency) } \\
\hline Less than 5 years & $10 \%(n=2)$ & $10 \%(n=2)$ \\
\hline $5-10$ years & $35 \%(n=7)$ & $65 \%(n=13)$ \\
\hline $10-15$ years & $15 \%(n=3)$ & $15 \%(n=3)$ \\
\hline $15-20$ years & $10 \%(n=2)$ & $10 \%(n=2)$ \\
\hline $20-25$ years & $30 \%(n=6)$ & $15 \%(n=3)$ \\
\hline
\end{tabular}

Average number of cigarette/ tobacco/ beedi/ areca nut consumed, percentage (frequency)

\begin{tabular}{l|c|c}
\hline Less than 5 years & $10 \%(n=2)$ & $15 \%(n=3)$ \\
\hline $5-10$ years & $65 \%(n=13)$ & $45 \%(n=9)$ \\
\hline $10-15$ years & $15 \%(n=3)$ & $20 \%(n=4)$ \\
\hline $15-20$ years & $10 \%(n=2)$ & $20 \%(n=4)$ \\
\hline
\end{tabular}

Frequency of use, percentage (frequency)

\begin{tabular}{|l|c|c|}
\hline Less than 5 times & $35 \%(n=7)$ & $65 \%(n=13)$ \\
\hline 5 -10 times & $60 \%(n=12)$ & $25 \%(n=5)$ \\
\hline $10-15$ times & $5 \%(n=1)$ & $10 \%(n=2)$ \\
\hline \begin{tabular}{l} 
Duration of use, percentage (frequency) \\
\hline 5 -10 minutes
\end{tabular} & $70 \%(n=14)$ & $80 \%(n=16)$ \\
\hline $10-20$ minutes & $30 \%(n=6)$ & $20 \%(n=4)$ \\
\hline \begin{tabular}{l} 
Site of placement of smokeless tobacco, percentage (frequency) \\
\hline Buccal mucosa
\end{tabular} & $60 \%(n=12)$ & $65 \%(n=13)$ \\
\hline Floor of mouth & $10 \%(n=2)$ & $25 \%(n=5)$ \\
\hline Labial vestibule & $30 \%(n=6)$ & $10 \%(n=2)$ \\
\hline
\end{tabular}

TABLE 2. Mean difference between study groups in MMP-9 values

\begin{tabular}{|c|c|c|c|}
\hline Groups & Number & Mean \pm SD & $p$-value \\
\hline Group A (OSMF) & 20 & $9.42 \pm 2.882$ & \multirow[t]{3}{*}{$<0.001^{*}$} \\
\hline Group B (leukoplakia) & 20 & $10.59 \pm 2.862$ & \\
\hline Group C (control) & 20 & $2.96 \pm 2.378$ & \\
\hline
\end{tabular}

and $2.96 \mathrm{ng} / \mathrm{ml} \pm 2.378$, respectively (Table 2 ). The mean MMP-9 level was higher in group B $(10.59 \mathrm{ng} / \mathrm{ml})$ when compared to group A $(9.42 \mathrm{ng} / \mathrm{ml})$ and group C $(2.96 \mathrm{ng} / \mathrm{ml})$ (one-way ANOVA with post-hoc test; 
TABLE 3. Paired comparisons between study groups in MMP-9 values

\begin{tabular}{|l|c|c|c|}
\hline \multicolumn{2}{|l|}{ Dependent groups (I)/Study group (J) } & Sean difference & Standard error \\
\hline \begin{tabular}{l} 
Group A (OSMF) \\
\hline Leukoplakia
\end{tabular} & -1.173 & .859 & 0.532 \\
\hline Control & $6.460^{*}$ & .859 & $<0.001^{*}$ \\
\hline \begin{tabular}{l} 
Group B (leukoplakia) \\
\hline OSMF
\end{tabular} & 1.173 & .859 & 0.532 \\
\hline Control & $7.633^{*}$ & .859 &.$<0.001^{*}$ \\
\hline Group C (control) & & & $<0.001^{*}$ \\
\hline OSMF & $-6.460^{*}$ & .859 & $<0.001^{*}$ \\
\hline Leukoplakia & $-7.633^{*}$ & .859 & \\
\hline
\end{tabular}

$p<0.05$ is significant; Analysis used: Bonferroni-adjusted one-way ANOVA test

$p<0.001)$. Bonferroni-adjusted one-way ANOVA was performed and the obtained $p$-value was highly significant when comparing group A with group $\mathrm{C}(p<0.001)$, and group B with group $\mathrm{C}(p<0.001)$ (Table 3$)$.

Considering variants of leukoplakia, homogeneity occurred in $70 \%(n=14)$, while non-homogenous leukoplakia occurred in $30 \%(n=6)$ of the participants. Specked or proliferative verrucous types were not found in the current study. The lesions were categorized based on the degree of dysplasia; however, leukoplakia with mild $(10.47 \pm 1.08)$ and moderate $(10.60 \pm 1.48)$ dysplasia showed no significant differences (unpaired $t$-test, $p=0.901)$ in terms of MMP-9.

Receiver operating characteristic curve (ROC) were plotted, including group A versus group $\mathrm{C}$ and group $B$ versus group $C$. The area under the curve (AUC) for group A versus group C $(p=0.948)$ and group B versus group $C(p=0.975)$ was found statistically insignificant (Figures 2A, B).

A

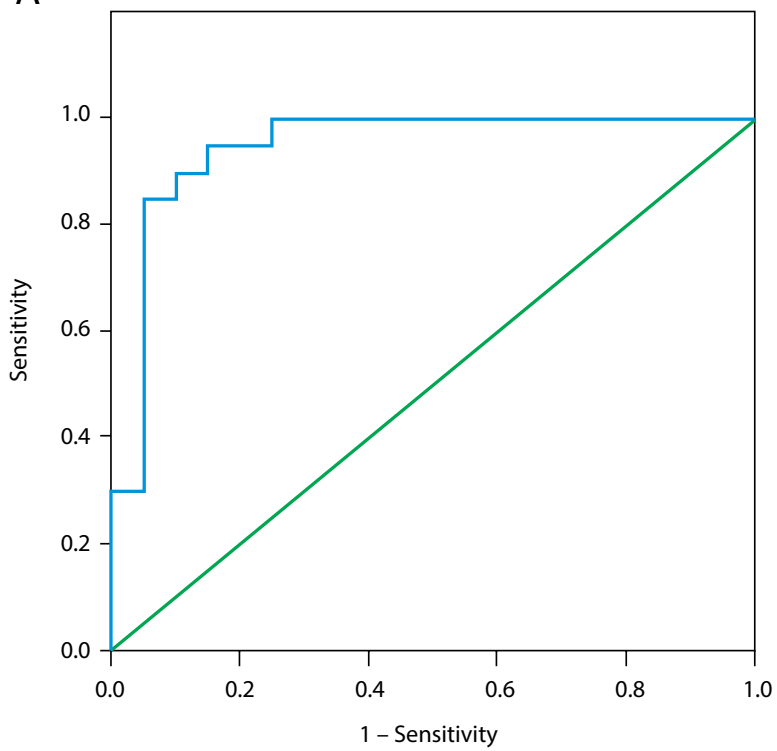

\section{DISCUSSION}

Oral squamous cell carcinoma (OSCC) is the most common type of cancer occurring in the oral cavity. It is often diagnosed in advanced stages, leading to poor prognosis and low survival rates [2]. An early diagnosis can benefit in $80 \%$ survival rates, but there seems to be an evergrowing struggle to identify suitable biological indicators for characterization and prediction of malignant transformation $[7,8]$.

There is a need to identify sensitive biomarkers for screening of OSCC and OPMD in early stages in order to deliver timely treatment. In this context, MMPs were shown of considerable importance $[2,8]$. MMPs are secreted by neutrophils, macrophages, or fibroblasts on the stimulus of transforming growth factor $\beta$ (TGF- $\beta$ ) and interleukin-8 (IL-8), and have ability to degrade extracellular matrix. MMP sustains bioavailability of growth factors, thus aiding in the proliferation of cancerous cells [2].

B

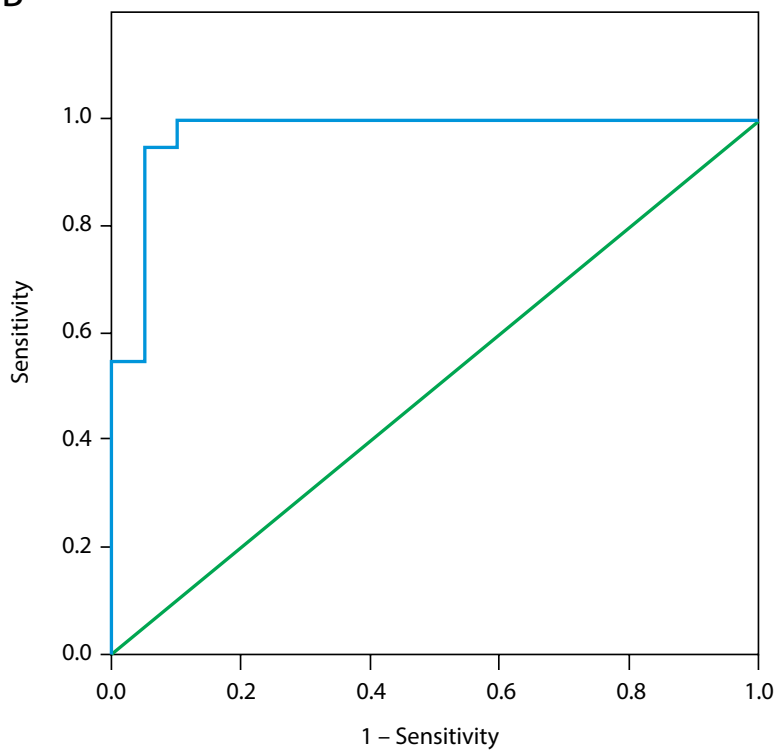

FIGURE 2. A) ROC for group A vs. group C. B) ROC for group B vs. group C 
Studies have shown that consumed in various tobacco forms surface alterations and keratinization in oral $\mathrm{mu}$ cosa corresponding to OPMDs or early OSCC. These differences lead to alteration of MMP-9 secretion [2]. This change in MMP-9 concentration can be detected in serum or saliva of subjects even with OPMD. Therefore, the current study was designed to detect MMP-9 levels in common OPMDs, including OSMF and oral leukoplakia.

In the current study, the mean age of participants with OSMF was $50.30 \pm 9.96$ years, with peak occurrence in the age group of 40-49 years. These observations were in accordance with previous studies $[2,11]$, who reported mean age of study participants with OSMF as 44.00 \pm 14.19 years. The possible reason for OSMF occurring in middle-aged individuals could be a result of expanded social experiences and financial freedom they acquire at this age, and that they enjoy chewing betel nut, gutka, and container masala to overcome stress related to lifestyle changes or peer pressure [12]. Numerous studies [13-15] reported mean age of participants to be between 20 and 30 years. This observation could be attributed to the ease of availability of betel nut, gutka at school, college canteens, and confectionary shops, and associated euphoric effect of areca nut with its' addictive property [13]. In case of oral leukoplakia, the age distribution ranged from 20 and 60 years, with a mean age of $46.70 \pm$ 12.59 years, with a peak occurrence of oral leukoplakia in 40-49-year-olds. This is in accordance with previous studies [16-19], with mean age of study participants of 50 years and above. The occurrence of oral leukoplakia in this age group could be ascribed to the ease of availability of cigarettes as well as the urge of smoking, cue-driven smoking urges, or situational cravings [20].

The gender distribution for OSMF was reported as $60 \%$ and $40 \%$ between males and females, which was in accordance with studies conducted by Shivam et al. and Kumar et al. [15, 21]. Similarly for oral leukoplakia, gender distribution was recorded as $95 \%$ and $5 \%$ between males and females as reported by Bisht et al. and Gopinath et al. [22, 23], respectively.

This study observed a statistically significant difference in MMP-9 levels of group A and group B patients, when compared to controls of group C. Similar observations were reported by Rajendran et al. [24]. In their study, the excessive collagenization taking place in OSMF could set in motion a regulatory reflex mechanism, causing an up regulation in the matrix enzyme expression, which was attributable for the raise of MMP-9 level. The current observations are also consistent with those reported by Vajaria et al. [25], where statistically significant increase of MMP-9 levels was observed in oral cancer and pre-cancers, as compared to controls. The elevated level of salivary MMP-9 in patients with oral PMDs and oral cancer may be important for assessing early changes occurring during neoplastic transformation of oral cancer.

The findings of the current study are also in line with a research conducted by Ghallab et al., who showed a sta- tistically significant increase of serum and salivary MMP9 levels and chemerin biomarker in patients with OSCC and OPMDs, when compared with controls. ROC analysis showed higher sensitivity and specificity compared with serum MMP-9 [26]. A recent study by Smriti et al. [2] reported statistically significant increase in MMP-9 levels $(p<0.001)$ in patients with OPMD'S and OSCC compared with healthy controls and tobacco chewers. Expressions of MMP-9 levels were established as a diagnostic marker of oral cancer tissue, and over-expression of MMP-9 could be an indicator of malignant transformation or progression of staging [27].

The findings of the present study are encouraging for using MMP-9 as a diagnostic marker in estimating and monitoring high-risk groups prone to develop OPMDs. Utilizing this marker as a part of routine clinical investigation can play an essential role to identify individuals who are genetically predisposed (family history of oral cancers or PMDs, like xeroderma pigmentosa or epidermolysis bullosa), and who are not exhibiting any morphological variation or lesion in oral cavity. The strength of the study was reflected in the highly significant statistical value obtained. However, a larger sample size is recommended to further validate the results. A future prospect of the current study would lie in further investigating of the expression of MMP-9 levels in different types of oral leukoplakia and various stages of OSMF.

\section{CONCLUSIONS}

The observations of the current study report strong expression of MMP-9 in oral leukoplakia group compared to OSMF. However, the expression was showed a minimum in the control group. The results strongly support MMP-9 as a reliable biomarker in accessing malignant transformation of OPMDs. This investigation should be considered as a vital component in screening patients with a habit related to tobacco, and those genetically predisposed to oral cancer.

\section{ACKNOWLEDGEMENT}

The authors would like to thank to Ezhumalai G, senior statistician and research consultant, MGMCRI. Dr. Balanehru Subramanian, Director of Central InterDisciplinary Research Facility (CIDRF) for helping avail facilities from CIDRF and DBT-funded project (BT/INF/ 22/SP17432/2016) - Center for Animal Research Training and Services (CAReTS).

\section{CONFLICT OF INTEREST}

The authors declare no potential conflicts of interest with respect to the research, authorship, and/or publication of this article. 


\section{References}

1. Warnakulasuriya S, Johnson NW, van der Waal I. Nomenclature and classification of potentially malignant disorders of the oral mucosa. J Oral Pathol Med 2007; 36: 575-580.

2. Smriti K, Ray M, Chatterjee T, et al. Salivary MMP-9 as a biomarker for the diagnosis of oral potentially malignant disorders and oral squamous cell carcinoma. Asian Pac J Cancer Prev 2020; 21: 233-238.

3. Napier SS, Speight PM. Natural history of potentially malignant oral lesions and conditions: an overview of the literature. J Oral Pathol Med 2008; 37: 1-10.

4. Martorell-Calatayud A, Botella-Estrada R, Bagan-Sebastian JV, et al. Oral leukoplakia: clinical, histopathological, and molecular features and therapeutic approach. Actas Dermosifiliogr 2009; 100: 669-684.

5. Rao NR, Villa A, More CB, et al. Oral submucous fibrosis: a contemporary narrative review with a proposed inter-professional approach for an early diagnosis and clinical management. J Otolaryngol Head Neck Surg 2020; 49: 3.

6. Nigam NK, Aravinda K, Dhillon M, et al. Prevalence of oral submucous fibrosis among habitual gutkha and areca nut chewers in Moradabad district. J Oral Biol Craniofac Res 2014; 4: 8-13.

7. Shih YH, Wang TH, Shieh TM, Tseng YH. Oral submucous fibrosis: a review on etiopathogenesis, diagnosis, and therapy. Int J Mol Sci 2019; 20: 2940.

8. Feller L, Lemmer J. Oral squamous cell carcinoma: epidemiology, clinical presentation and treatment. J Cancer Ther 2012; 3: 263-268.

9. Hema Shree K, Ramani P, Sherlin H, et al. Saliva as a diagnostic tool in oral squamous cell carcinoma - a systematic review with meta analysis. Pathol Oncol Res 2019; 25: 447-453.

10. Yadav L, Puri N, Rastogi V, Satpute P, Ahmad R, Kaur G. Matrix metalloproteinases and cancer-roles in threat and therapy. Asian Pac J Cancer Prev 2014; 15: 1085-1091.

11. Siriwardena BS, Jayawardena KL, Senarath NH, Tilakaratne WM. An evaluation of clinical and histopathological aspects of patients with oral submucous fibrosis in the background of oral squamous cell carcinoma. BioMed Res Int 2018; 2018: 4154165.

12. Goel S, Ahmed J. A comparative study on efficacy of different treatment modalities of oral submucous fibrosis evaluated by clinical staging in population of Southern Rajasthan. J Can Res Ther 2015; 11: 113-118

13. Hosein M, Mohiuddin S, Fatima N. Association between grading of oral submucous fibrosis with frequency and consumption of areca nut and its derivatives in a wide age group: a multi-centric cross sectional study from Karachi, Pakistan. J Cancer Prev 2015; 20: 216-222.

14. Kumar S. Oral submucous fibrosis: a demographic study. J Indian Acad Oral Med Radiol 2016; 28: 124-128.

15. Shivam AK, Azam F, Sadiq H. Prevalence of oral submucous fibrosis among habitual gutkha and areca nut chewers in Dhanbad district. Int J Prev Clin Dent Res 2018; 5: 60-62.

16. Liu W, Wang YF, Zhou HW, Shi P, Zhou ZT, Tang GY. Malignant transformation of oral leukoplakia: a retrospective cohort study of 218 Chinese patients. BMC Cancer 2010; 10: 685.

17. Birur P, Shubhasini AR, Bhanushree R, Mendonca P. Correlation of oral homogenous leukoplakia with grades of oral epithelial dysplasia. IOSR Journal of Dental and Medical Sciences 2014; 13: 98-103.

18. Starzynska A, Pawlowska A, Renkielska D, et al. Estimation of oral leukoplakia treatment records in the research of the Department of Maxillofacial and Oral Surgery, Medical University of Gdansk. Postep Dermatol Alergol 2015; 32: 114-122.

19. Vila PG, Pérez-Sayáns M, Peñaranda JM, et al. Survival study of leukoplakia malignant transformation in a region of northern Spain. Med Oral Patol Oral Cir Bucal 2018; 23: e413-e420.

20. West R. Tobacco smoking: health impact, prevalence, correlates and interventions. Psychol Health 2017; 32: 1018-1036.

21. Kumar KK, Saraswathi TR, Ranganathan K, Devi MU, Elizabeth J. Oral submucous fibrosis: a clinico-histopathological study in Chennai. Indian J Dent Res 2007; 18: 106-111.
22. Bisht RS, Singh AK, Sikarwar V, Darbari A. Study over the clinical picture and histopathology of leukoplakia and to establish the correlation between causative factors in the patients of Garhwal hill region. Natl J Maxillofac Surg 2013; 4: 177-180.

23. Gopinath D, Thannikunnath BV, Neermunda SF. Prevalence of carcinomatous foci in oral leukoplakia: a clinicopathologic study of 546 Indian samples. J Clin Diagn Res 2016; 10: ZC78-83.

24. Rajendran R, Mohammed PR, Shaikh S, Pillai MR. Expression of matrix metalloproteinases (MMP-1, MMP-2 and MMP-9) and their inhibitors (TIMP-1 and TIMP-2) in oral submucous fibrosis. Indian J Dent Res 2006; 17: 161-166.

25. Vajaria BN, Patel KR, Begum R, Shah FD, Patel JB, Joshi GM, Patel PS. Clinical significance of salivary matrix metalloproteinase- 9 in oral precancerous conditions and oral cancer. Cancers Rev 2014; 1: 33-44.

26. Ghallab NA, Shaker OG. Serum and salivary levels of chemerin and MMP-9 in oral squamous cell carcinoma and oral premalignant lesions. Clin Oral Invest 2017; 21: 937-947.

27. Venugopal A, Uma Maheswari T. Expression of matrix metalloproteinase- 9 in oral potentially malignant disorders: a systematic review. J Oral Maxillofac Pathol 2016; 20: 474. 